ISSN 1063-7737, Astronomy Letters, 2008, Vol. 34, No. 10, pp. 686-698. (C)Pleiades Publishing, Inc., 2008. Original Russian Text (C)V.V. Bobylev, 2008, published in Pis'ma v Astronomicheskĩ Zhurnal, 2008, Vol. 34, No. 10, pp. 757-770.

\title{
Open Clusters IC 4665 and Cr 359 and a Probable Birthplace of the Pulsar PSR B1929+10
}

\author{
V.V. Bobylev \\ Central (Pulkovo) Astronomical Observatory of RAS, St-Petersburg
}

\begin{abstract}
Based on the epicyclic approximation, we have simulated the motion of the young open star clusters IC 4665 and Collinder 359. The separation between the cluster centers is shown to have been minimal $7 \mathrm{Myr}$ ago, $36 \mathrm{pc}$. We have established a close evolutionary connection between IC 4665 and the Scorpius-Centaurus association - the separation between the centers of these structures was $\approx 200$ pc 15 Myr ago. In addition, the center of IC 4665 at this time was near two well-known regions of coronal gas: the Local Bubble and the North Polar Spur. The star HIP 86768 is shown to be one of the candidates for a binary (in the past) with the pulsar PSR B1929+10. At the model radial velocity of the pulsar $V_{r}=2 \pm 50 \mathrm{~km} \mathrm{~s}^{-1}$, a close encounter of this pair occurs in the vicinity of IC 4665 at a time of $-1.1 \mathrm{Myr}$. At the same time, using currently available data for the pulsar B1929+10 at its model radial velocity $V_{r}=200 \pm 50 \mathrm{~km} \mathrm{~s}^{-1}$, we show that the hypothesis of Hoogerwerf et al. (2001) about the breakup of the $\zeta$ Oph-B1929+10 binary in the vicinity of Upper Scorpius (US) about $0.9 \mathrm{Myr}$ ago is more plausible.
\end{abstract}

\section{INTRODUCTION}

At present, fairly reliable estimates of such parameters as the distance and proper motion components are available for a number of nearby neutron stars, but as yet there is no information about their radial velocities. In the problem of determining the possible birthplace of a neutron star, the radial velocity is a free parameter.

Based on the Hipparcos Catalogue and radioastrometric data, Hoogerwerf et al. (2001) analyzed 56 nearby high-velocity stars $(r<700 \mathrm{pc})$, the so-called runaway stars, to determine their possible birthplace inside nearby young stellar groups - open star clusters or associations. One of their conclusions was that the star $\zeta$ Oph and the pulsar PSR B1929+10 (which, according to the J2000 coordinate system, was designated as PSR J1932+1059) originated jointly in a supernova explosion in a binary about 1 Myr ago in the part of the Scorpius-Centaurus association designated as US (Upper Scorpius).

However, subsequent VLBI measurements of the parallax for PSR B1929+10 (Brisken et al. 2002; Chatterjee et al. 2004) showed that the probable distance to the pulsar differs significantly from that used by Hoogerwerf et al. (2001). In the opinion of Chatterjee et al. (2004), the new data basically reject the hypothesis of Hoogerwerf et al. (2001) about the birthplace of PSR B1929+10 at the boundary of the Scorpius-Centaurus association and its 
connection with the star $\zeta$ Oph. In this paper, we test Hoogerwerf's hypothesis using new observational data. In addition, estimating the probability of other hypotheses is also of considerable interest.

One of such hypotheses was proposed by Walter (2001), who suggested considering a different neutron star as a binary (in the past) with $\zeta$ Oph, namely, RX J185635-3754.

For our part, we also suggest considering the hypothesis that the birthplace of PSR B1929+10 could be the open cluster IC 4665 or Cr 359. The idea of this hypothesis is based on the simulations of the PSR B1929+10 trajectory over the celestial sphere (Fig. 2 from Hoogerwerf et al. (2001)) at various radial velocities $\left(+200\right.$ or $\left.-200 \mathrm{~km} \mathrm{~s}^{-1}\right)$, which showed that it passed in the immediate vicinity of the open clusters IC 4665 and $\mathrm{Cr} 359$. In this paper, we determine the radial velocity of the pulsar at which its encounter with these clusters is closest.

In the immediate solar neighborhood with a radius of 200-300 pc, we know such regions of interstellar tenuous hot gas with a temperature $T \approx 10^{6} \mathrm{~K}$ as the Local Bubble and the North Polar Spur. Since the Local Bubble is a compact region that is almost free from absorbing matter, it was first discovered by Fitzgerald (1968) through an analysis of the interstellar reddening of stars. The absorption maps of the distribution of neutral gas in NaI lines constructed by Sfeir et al. (1999) show an inclination to the Galactic plane as for the Gould Belt. An overview of the formation scenarios for the North Polar Spur can be found in Miller et al. (2007). Data on all of the known bubbles in the solar neighborhood were collected in Heiles (1998).

The physical processes that produce bubbles, in outline, are as follows. Multiple supernova explosions occur in young open clusters. This gives rise to stellar winds and shock waves that sweep out the gas to the periphery of the local region in question, where it produces blobs in the form of shells or extended walls. At the shell boundaries, the gas density increases significantly, the gas cools down, and molecular clouds are formed. During supernova explosions, the gas inside the shell can heat up and radiate in the X-ray range. In the opinion of Berghöfer and Breitschwerdt (2002), the most realistic theory for the origin of the Local Bubble is the hypothesis about multiple, but not simultaneous explosions of about 20 supernovae during the past 10-20 Myr. Frish (1995), Maiz-Apellániz (2001), and Breitschwerdt and de Avillez (2006) substantiate the viewpoint that supernova explosions in the Scorpius-Centaurus association, which is part of the Gould Belt, are the most suitable objects responsible for the formation of both the Local Bubble and the North Polar Spur.

The open clusters IC 4665 and Cr 359 have a high probability of belonging to the Gould Belt both in their age and spatial position (Piskunov et al. 2006) and in kinematics (Bobylev 2006). Since there are very few young open star clusters in the first Galactic quadrant that are members of the Gould Belt and that have reliable kinematic data (particularly the radial velocities), studying the trajectories of the open clusters IC 4665 and Cr 359 and members of the Scorpius-Centaurus association is of great importance for understanding the evolution of the Gould Belt.

The goal of this paper is to establish the evolutionary connections of the young open clusters IC 4665 and Cr 359 with the structure of the Gould Belt, to establish the possible influence of the clusters on the bubble development, and to determine the most probable birthplace of the pulsar PSR B1929+10 by varying its radial velocity.

The problems posed here are solved by constructing the trajectories of the objects using the epicyclic approximation. 


\section{THE METHOD}

In this paper, we use a rectangular Galactic coordinate system with the axes directed away from the observer toward the Galactic center $\left(l=0^{\circ}, b=0^{\circ}\right.$, the $X$ axis), along the Galactic rotation $\left(l=90^{\circ}, b=0^{\circ}\right.$, the $Y$ axis $)$, and toward the North Galactic Pole $\left(b=90^{\circ}\right.$, the $Z$ axis). The corresponding space velocity components of the objects $U, V, W$ are also directed along the $X, Y, Z$ axes (Kulikovskii 1985).

The method of the epicyclic approximation (Lindblad 1927, 1959) allows the orbits of stars to be constructed in a coordinate system rotating around the Galactic center in a circular orbit.We assume that the origin of the coordinate system coincides with the local standard of rest and that the stars move along epicycles in the direction opposite to the Galactic rotation. We use the method in a form associated with a rectangular coordinate system (Fuchs et al. 2006):

$$
\begin{gathered}
X(t)=X(0)+\frac{U(0)}{\kappa} \sin (\kappa t)+\frac{V(0)}{2 B}(1-\cos (\kappa t)), \\
Y(t)=Y(0)+2 A\left(X(0)+\frac{V(0)}{2 B}\right) t-\frac{\Omega_{0}}{B \kappa} V(0) \sin (\kappa t)+\frac{2 \Omega_{0}}{\kappa^{2}} U(0)(1-\cos (\kappa t)), \\
Z(t)=\frac{W(0)}{\nu} \sin (\nu t)+Z(0) \cos (\nu t),
\end{gathered}
$$

where $t$ is the time in $\operatorname{Myr}\left(\mathrm{pc} / \mathrm{Myr}=0.978 \mathrm{~km} \mathrm{~s}^{-1}\right)$, which is measured into the past; $\kappa=$ $\sqrt{-4 \Omega_{0} B}$ is the epicyclic frequency; $\mathrm{A}$ and $\mathrm{B}$ are the Oort constants; $\Omega_{0}$ is the angular velocity of Galactic rotation of the local standard of rest, $\Omega_{0}=A-B$; and $\nu=\sqrt{4 \pi G \rho_{0}}$ is the frequency of the vertical oscillations, where $G$ is the gravitational constant and $\rho_{0}$ is the star density in the solar neighborhood. The space velocities of the objects are calculated for any necessary time using the formulas

$$
\begin{gathered}
U(t)=U(0) \cos (\kappa t)-\frac{\kappa}{-2 B} V(0) \sin (\kappa t), \\
V(t)=\frac{-2 B}{\kappa} U(0) \sin (\kappa t)+V(0) \cos (\kappa t), \\
W(t)=W(0) \cos (\nu t)-Z(0) \nu \sin (\nu t) .
\end{gathered}
$$

The parameters $X(0), Y(0), Z(0)$ and $U(0), V(0), W(0)$ in Eqs. (1) and (2) denote the current positions and velocities of the objects. The velocities $U, V, W$ are given relative to the local standard of rest with $(U, V, W)_{L S R}=(10.00,5.25,7.17) \pm(0.36,0.62,0.38) \mathrm{km} \mathrm{s}^{-1}$ (Dehnen and Binney 1998).

Following Fuchs et al. (2006), we adopted $\rho_{0}=0.1 M_{\odot} \mathrm{pc}^{-3}$, which gives $\nu=0.074 \mathrm{~km}$ $\mathrm{s}^{-1} \mathrm{pc}^{-1}$. We also took the Oort constants $A=13.7 \pm 0.6 \mathrm{~km} \mathrm{~s}^{-1} \mathrm{kpc}^{-1}$ and $B=-12.9 \pm$ $0.4 \mathrm{~km} \mathrm{~s}^{-1} \mathrm{kpc}^{-1}$ that we found previously (Bobylev 2004) by analyzing the independent determinations of these parameters by various authors; in that case, $\kappa=0.037 \mathrm{~km} \mathrm{~s}^{-1} \mathrm{pc}^{-1}$.

\section{THE DATA}

The necessary input data on the objects under consideration, such as their equatorial coordinates, proper motion components, radial velocities, and parallaxes, are given in Table 1. 
Table 2 gives the spatial coordinates $X(0), Y(0), Z(0)$ and space velocities $U(0), V(0), W(0)$ of the stars and clusters under consideration.

IC 4665 and Cr 359. For the open cluster IC 4665, we use the coordinates of the center, proper motion components, and an estimate of the photometric distance $r=352 \pm 70 \mathrm{pc}$ from the CRVOCA Catalog (Kharchenko et al. 2007) and the radial velocity from Manzi et al. (2007), where it was determined using 39 most probable cluster members. According to Manzi et al. (2007), the age of IC 4665 is $27.7_{-3.5}^{+4.2} \mathrm{Myr}$. This estimate is interesting in that it depends little on the quality of isochrones, because it was obtained by comparing the lithium abundances in cluster stars with such well-known young clusters as NGC 2547 and IC 2391. Other authors give the following "isochronic" cluster age estimates: $36 \mathrm{Myr}$ (Mermilliod 1981), 30-100 Myr (Prosser 1993), and 43 Myr (Piskunov et al. 2006).

For the open cluster Cr 359, we use the coordinates of the center, proper motion components, and radial velocity from the CRVOCA Catalog (Kharchenko et al. 2007) and the distance $r=450 \pm 200 \mathrm{pc}$ from Lodieu et al. (2006), who conducted a critical review of the estimates obtained by various authors. According to Lodieu et al. (2006), the age of Cr 359 is $60 \pm 20 \mathrm{Myr}$. Other authors estimate this cluster as a younger one, 32 Myr (Piskunov et al. 2006) and $\approx 30$ Myr (Wielen 1971; Abt and Cardona 1983).

According to Kharchenko et al. (2007), the apparent radii of IC 4665 and Cr 359 are $1 .^{\circ} 0$ and $1 .^{\circ} 1$, respectively.

de Wit et al. (2006) estimated the mass of IC 4665 to be 300-350 $M_{\odot}$. Cr 359 probably has a similar mass.

PSR B1929+10. The radio pulsar PSR B1929+10 is an isolated neutron star with an age of $3 \mathrm{Myr}$ (estimated from the ratio $\mathrm{P} / 2 \dot{\mathrm{P}}$ and belongs to the population of nearby neutron stars that are closely related to the Gould Belt (Popov et al. 2003; Motch et al. 2006).

For PSR B1929+10, we use the data from Chatterjee et al. (2004) that were obtained from VLBI measurements. The most important refined parameter is the new parallax of the pulsar, $\pi=2.77 \pm 0.07$ mas.

Note that Hoogerwerf et al. (2001) adopted a model value of $\pi=4 \pm 2$ mas. The preliminary distance estimates for the pulsar that made it closer to the Sun served as a basis for this choice.

US, UCL, and LCC. The initial positions of the centers $(X(0), Y(0), Z(0))=$ $(134,-20,52) \mathrm{pc}$ and heliocentric velocities $(U(0), V(0), W(0))=(-6.7,-16.0,-8.0) \pm$ $(5.9,3.5,2.7) \mathrm{km} \mathrm{s}^{-1}$ for US, $(119,-67,31) \mathrm{pc}$ and $(-6.8,-19.3,-5.7) \pm(4.6,4.7,2.5) \mathrm{km}$ $\mathrm{s}^{-1}$ for UCL, $(62,-100,10) \mathrm{pc}$ and $(-8.2,-18.6,-6.4) \pm(5.1,7.3,2.6) \mathrm{km} \mathrm{s}^{-1}$ for LCC were taken from Fernández et al. (2006). These authors obtained the positions of the centers (with errors of $1.2 \mathrm{pc}$ ) using data from de Zeeuw et al. (1999); the velocities were determined by Sartori et al. (2003) based on the space velocities of a large number of stars (more than 120 for each of the groups). Fernández et al. (2006) used these data to analyze the kinematics of the members of the Scorpius. Centaurus association. However, the orbits of the objects were calculated using a model that, apart from the axisymmetric Galactic potential, also included the potential from a spiral density wave and the potential of the bar at the Galactic center (Fernández et al. 2008). Comparing the results obtained by various methods is of considerable interest. 


\section{RESULTS}

\section{The Neighborhoods of IC 4665, Cr 359, and PSR B1929+10}

The encounters of the centers. Based on Eqs. (1) and (2), we obtained several solutions at various radial velocities of the pulsar PSR B1929+10, $V_{r}$ (PSR). The results are the following:

(1) at $V_{r}(\mathrm{PSR})=+45 \mathrm{~km} \mathrm{~s}^{-1}$, the pulsar encounters with the cluster IC 4665 at the distance $\Delta_{r}=\sqrt{\Delta X^{2}+\Delta Y^{2}+\Delta Z^{2}}=52$ pc at $t=-0.85 \mathrm{Myr}$;

(2) at $V_{r}(\mathrm{PSR})=-60 \mathrm{~km} \mathrm{~s}^{-1}$, the minimum distance from the pulsar to the center of Cr 359 is $\Delta_{r}=38$ pc at $t=-1.0 \mathrm{Myr}$;

(3) at $V_{r}(\mathrm{PSR})=0 \mathrm{~km} \mathrm{~s}^{-1}$, we obtain a joint encounter with the two clusters. In this case, the minimum distances from the pulsar to the centers of Cr 359 and IC 4665 are $\Delta_{r}=69 \mathrm{pc}$ and $\Delta_{r}=60 \mathrm{pc}$, respectively, at $t=-1.0 \mathrm{Myr}$.

As we see, the pulsar trajectory can pass through the coronas of the two clusters under consideration. As was noted by de Wit et al. (2006), the tidal radius of IC 4665 is $\approx 1^{\circ}$. At an assumed distance to the cluster of $0 r=352 \mathrm{pc}$, it is $\approx 6 \mathrm{pc}$. However, the stars with a common proper motion occupy an area of $\approx 100$ square degrees on the celestial sphere (Lodieu et al. 2006) and, hence, the coronal radius of IC 4665 is $\approx 30$ pc. As can be seen from Table 2, the error in the spatial position of the center of IC 4665 is about $\pm 50 \mathrm{pc}$; its spatial displacement in 2 Myr is insignificant.

In this respect, $\mathrm{Cr} 359$ is of lesser interest, since the error in its distance is $44 \%$. Therefore, the radius of its corona lies within the range $20-60$ pc, but the spatial localization accuracy of the cluster is low, about $\approx 150$ pc (Table 2 ).

Next, we take the next natural step or, more specifically, we search for a suitable rapidly flying star, a possible member of a binary. For this purpose, we use the list of such stars from Hoogerwerf et al. (2001). We can see from Fig. 2 in Hoogerwerf et al. (2001) that the trajectories of three stars passed in the immediate vicinity of IC 4665 and Cr 359 on the celestial sphere with a radius of $\approx 15^{\circ} 2$ Myr ago: HIP 66524, HIP 86768, and HIP 91599. To calculate the space velocities, we invoke the current radial velocities of these stars from the Pulkovo Catalog of Radial Velocities (Gontcharov 2006).

The construction of the trajectories for the three rapidly flying stars shows that HIP 86768 is most interesting among them.

Indeed, HIP 66524 moves away fairly rapidly toward the Galactic center in $X$ coordinate, without having a close encounter with the objects of interest to us.

For HIP 91599 (star No. 20 from the list of Hoogerwerf et al. 2001), the situation is slightly different. At the radial velocity of the pulsar PSR B1929+10 $V_{r}(\mathrm{PSR})=+250 \mathrm{~km}$ $\mathrm{s}^{-1}$, it encounters with HIP 91599 at the distance $\Delta_{r}=47 \mathrm{pc}$ at $t=-0.5 \mathrm{Myr}$. The modulus of the velocity difference between the pulsar and HIP 91599 at the time of their encounter is $\Delta_{V}=\sqrt{\Delta U^{2}+\Delta V^{2}+\Delta W^{2}}=316 \mathrm{~km} \mathrm{~s}^{-1}$.

At the radial velocity of the pulsar $V_{r}(\mathrm{PSR})=+2 \mathrm{~km} \mathrm{~s}^{-1}$, it encounters with HIP 86768 at the distance $\Delta_{r}=19 \mathrm{pc}$ at $t=-1.1 \mathrm{Myr}$. In this case, the modulus of the velocity difference between the pulsar and HIP 86768 at the time of their encounter $\Delta_{V}=206 \mathrm{~km}$ $\mathrm{s}^{-1}$. Taking into account the fairly large errors in the distances and velocities of HIP 86768, we can assume that a closer encounter is also possible. Therefore, simulating the encounters by taking into account the errors in the data is of great interest. 
Monte Carlo Simulations of Encounters. (1) Initially, we directly repeated the numerical experiment of Hoogerwerf et al. (2001) on the encounter between the star $\zeta$ Oph and PSR B1929+10. We computed 3 million orbits by taking into account the random errors in the input data, which were normally distributed with the $3 \sigma$ region. For the pulsar, we used old data: $V_{r}(P S R)=200 \pm 50 \mathrm{~km} \mathrm{~s}^{-1}, \pi=4 \pm 2$ mas, $\mu_{\alpha} \cos \delta=99 \pm 12(6 \times 2)$ mas $\mathrm{yr}^{-1}$, and $\mu_{\delta}=39 \pm 8(4 \times 2)$ mas $\mathrm{yr}^{-1}$ (following Hoogerwerf et al. (2001), we doubled the initial errors in the proper motions, as shown in parentheses). The results are: out of the 3 million orbits, 32505 encounters occur at distances $\Delta_{r} \leq 10 \mathrm{pc}(1.1 \%)$; in 4410 of the 32505 cases, the star and the pulsar were no farther than $10 \mathrm{pc}$ from the US center about $1 \mathrm{Myr}$ ago. The derived parameters are in excellent agreement with the results of Hoogerwerf et al. (2001).

(2) The same experiment on the encounter between $\zeta$ Oph and PSR B1929+10 with the currently available data for the pulsar: $V_{r}(\mathrm{PSR})=200 \pm 50 \mathrm{~km} \mathrm{~s}^{-1}, \pi=2.77 \pm 0.7(0.07 \times$ 10) mas, $\mu_{\alpha} \cos \delta=94.09 \pm 3.3(0.11 \times 30) \mathrm{mas} \mathrm{yr}^{-1}$, and $\mu_{\delta}=42.99 \pm 4.8(0.16 \times 30) \mathrm{mas} \mathrm{yr}^{-1}$. The initial values of the random errors (as shown in parentheses) were increased in order to have conditions comparable to those in experiment (1). The results are: out of the 3 million orbits, 74115 encounters occur at distances $\Delta_{r} \leq 10$ pc (2.5\%); in 5611 of the 74115 cases, the star and the pulsar were no father than $10 \mathrm{pc}$ from the US center about $1 \mathrm{Myr}$ ago. The domains of admissible values of $V_{r}, \pi, \mu_{\alpha} \cos \delta\left(\mu_{\alpha}^{*}\right)$, and $\mu_{\delta}$ at which 5611 encounters occur are presented in Fig. 1 for the star $\zeta$ Oph and in Fig. 2 for PSR B1929+10. Figure 3 shows the expected distribution F3D of minimum distance $\Delta_{r}$ calculated from the formula of Hoogerwerf et al. (2001),

$$
F_{3 D}\left(\Delta_{r}\right)=\frac{\Delta_{r}^{2}}{2 \sigma^{3} \sqrt{\pi}} \exp \left[-\frac{\Delta_{r}^{2}}{4 \sigma^{2}}\right]
$$

for the adopted $\sigma=2.5 \mathrm{pc}$.

(3) The experiment on the encounter between HIP 86768 and PSR B1929+10 for $V_{r}$ $(\mathrm{PSR})=2 \pm 50 \mathrm{~km} \mathrm{~s}^{-1}$ and the same values of $\pi=2.77 \pm 0.7(0.07 \times 10)$ mas, $\mu_{\alpha} \cos \delta=$

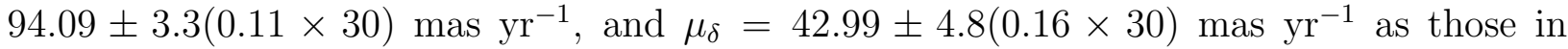
experiment (2). The results are: out of the 3 million orbits, 22708 encounters occur at distances $\Delta_{r} \leq 10 \mathrm{pc}(0.8 \%)$; in 6932 of the 22708 cases, the star and the pulsar were no father than $80 \mathrm{pc}$ from the center of IC 4665 about $1 \mathrm{Myr}$ ago. The domains of admissible values of $V_{r}, \pi, \mu_{\alpha} \cos \delta\left(\mu_{\alpha}^{*}\right)$, and $\mu_{\delta}$ at which 6932 encounters occur are presented in Fig. 4 for HIP 86768 and in Fig. 5 for PSR B1929+10. Figure 6 shows the expected distribution of minimum distance $\Delta_{r}$ calculated from Eq. (3) for the adopted $\sigma=6 \mathrm{pc}$.

(4) The experiment of the simulation of encounters between HIP 91599 and PSR $\mathrm{B} 1929+10$ at $V_{r}(\mathrm{PSR})=250 \pm 50 \mathrm{~km} \mathrm{~s}^{-1}$ shows that out of the 3 million orbits, 32961 encounters occur in the range $15 \mathrm{pc}<\Delta_{r} \leq 30 \mathrm{pc}\left(0\right.$ encounters at $\left.\Delta_{r} \leq 15 \mathrm{pc}\right)$ about 0.5 Myr ago. The stars HIP 86768 and HIP 91599 do not encounter at distances $\Delta_{r} \leq 10 \mathrm{pc}$, suggesting that the triple system HIP 86768 - HIP 91599-B1929+10 is unlikely.

(5) Testing the hypothesis of Walter (2001) - the experiment on the encounter between $\zeta$ Oph and the isolated neutron star (NS) RX J185635-3754 at $V_{r}(\mathrm{NS})=-50 \pm 50 \mathrm{~km}$ $\mathrm{s}^{-1}$. The results are: out of the 3 million orbits, 2144 encounters occur at distances $\Delta_{r} \leq$ $10 \mathrm{pc}(0.07 \%)$; there are no encounters with the US center at $\Delta_{r} \leq 10 \mathrm{pc}$. The derived characteristics are in good agreement with the results of Hoogerwerf et al. (2001). 


\section{IC 4665 and the Scorpius-Centaurus Association}

We traced the trajectories of IC 4665 and Cr 359 in a time interval comparable to their lifetime, up to $t=-30 \mathrm{Myr}$. For the mean values of the input parameters described in Section 2.1, we found the minimum separation between the cluster centers to be $\Delta_{r}=36 \mathrm{pc}$ at $t=-7$ Myr. Allowance for the errors in the cluster distances and velocities showed that the encounter time lies within the interval 0-12 Myr.

Figure 7 shows the positions of IC 4665, Cr 359, and members of the Scorpius-Centaurus association and their trajectories in the past $30 \mathrm{Myr}$; since the age of US does not exceed 5 Myr, the corresponding part of the trajectory is marked by the dotted line.

Figure 8 shows the calculated positions of IC 4665, the members of the ScorpiusCentaurus association UCL and LCC, and two bubbles, the Local Bubble (LB) and the North Polar Spur (L1) (Breitschwerdt and de Avillez 2006) at $t=-15$ Myr. The difference in the positions of the UCL and LCC centers at this time found from comparison with the data of Ortega et al. (2002) or Fernández et al. (2006) is $\approx \pm 50$ pc along the $X$ and $Y$ axes and very small, $\approx \pm 3 \mathrm{pc}$, along the $Z$ axis. We think these discrepancies attributable to differences in the models to be insignificant for the purposes of this paper. Note the approach of Fuchs et al. (2006), who simulated not the trajectories of the group centers, but the individual trajectories of very massive stars, potential supernovae, using Eq. (1). Some of them turned out to be near bubble L1 at $t=-15 \mathrm{Myr}$ (see Fig. 8). For IC 4665, apart from the coordinates of the center, Fig. 8 shows a cloud of 300 points (distributed with the $3 \sigma$ region) calculated by taking into account the errors in the input data for the cluster. As can be seen from the figure, the contribution from the errors in the distance is dominant.

\section{DISCUSSION}

\section{The Connection of IC 4665 and Cr 359 with the Scorpius-Centaurus Complex}

As we see from Fig. 7, the edge of the Gould Belt located in the first Galactic quadrant continuously approached the Galactic plane in a time interval of $\approx 30 \mathrm{Myr}$ in the past. This is in good agreement with the model for the evolution of the Gould Belt suggested by Olano (2001) and is consistent with the model calculations of the kinematic evolution of the Gould Belt performed by Perrot and Grenier (2003) using data on molecular clouds for the scenario with a "Galactic-plane crossing" (Fig. 7 from Perrot and Grenier (2003)), whereby the age of the Gould Belt is $51.8 \pm 1.0$ Myr. According to these two models, the edge of the Gould Belt located in the first Galactic quadrant reaches the Galactic plane at $\approx-10$ Myr. Our results show a similar motion. Thus, for example, the $Z$ coordinates of the centers of UCL and LCC (which lie in the plane of the Gould Belt) at $t=-15$ Myr fixed in Fig. 7 are close to $0 \mathrm{pc}$, while the $Z$ coordinates of the centers of IC 4665 and Cr 359 (rising above the plane of the Gould Belt) are close to $70 \mathrm{pc}$.

Most of the authors believe that the Scorpius-Centaurus association is part of the Gould Belt (de Zeeuw et al. 1999; Bobylev 2006). However, Fernández et al. (2008) advocate the point of view that the association could evolve independently of the Gould Belt (outside the scope of the supernova explosion hypothesis) and could be formed from the parent cloud that was compressed by collisions with a spiral density wave. 
On the other hand, whatever the origin of the association, the history of star formation in it agrees satisfactorily with the model of successive star formation suggested by Blaauw (1964; 1991) and developed by Preibish and Zinnecker (1999), as applied to US. The age estimates for the members of the Scorpius-Centaurus association (de Geus et al. 1989) lie within the ranges 5-6 Myr for US, 14-15 Myr for UCL, and 11-2 Myr for LCC. Both the present-day "isochronic" estimates (Mamajek et al. 2002; Sartori et al. 2003), 8-10 Myr for US and 16-20 Myr for UCL and LCC, and our kinematic age estimate for the entire association, 21 Myr (Bobylev and Bajkova 2007), agree well with these estimates.

Our results show that the separation between IC 4665 and the Scorpius-Centaurus association was much smaller in the past. Thus, for example, whereas the current separation between their centers is 302 pc (UCL-IC 4665), it was 215 pc 15 Myr ago and 120 pc 30 Myr ago. The minimum separation was $\approx 80 \mathrm{pc}$ at $-66 \mathrm{Myr}$, but our model approximation on such a long time scale is already unreliable. All of these facts suggest that they were formed from the same parent hydrogen cloud; the cluster IC 4665 has always been located on its periphery.

According to the model of successive star formation, the shocks from supernova explosions compress the nearby (within $\approx 100 \mathrm{pc}$ ) molecular clouds, triggering star formation in them. In our view, the role of IC 4665 in the evolution of the Scorpius-Centaurus association could lie in the fact that supernova explosions in this cluster could trigger star formation processes in the association itself.

At $t=-15 \mathrm{Myr}$, IC 4665 was near two well-known regions of coronal gas: the Local Bubble and the North Polar Spur.

Based on an analysis of HI data, Heiles (1998) obtained the following parameters for the North Polar Spur (which is known as the brightest component of radio loop I): the heliocentric distance to the center is $120 \mathrm{pc}$, the coordinates of the center are $l=320^{\circ}$ and $b=+5^{\circ}$, and the radius is $118 \mathrm{pc}$. According to the model of Willingale et al. (2003), it can be represented as an expanding spherical superbubble with the following parameters: the distance is $210 \mathrm{pc}$, the direction of the center is $l=352^{\circ}, b=+15^{\circ}$, and the bubble radius is 140 pc. X-ray observations of the North Polar Spur showed that the peak of emission with an energy of $3 / 4 \mathrm{keV}$ is observed in the direction with $l=26 .^{\circ} 8$ and $b=+22 .^{\circ} 0$ (Snowden et al. 1997; Willingale et al. 2003; Miller et al. 2007), i.e., at the bubble boundary. The current coordinates of IC 4665 are $l=30 .^{\circ} 6$ and $b=+17 .^{\circ} 1$. Taking into account the results shown in Figs. 7 and 8, we conclude that IC 4665 has always been located at a distance of 150-200 pc from the bubble boundary both in the past and at present. This means that supernova explosions in IC 4665 over the past several Myr could influence the formation of the North Polar Spur. This influence lies in the fact that supernova explosions in IC 4665 could produce a stellar counter-wind (the main explosions in the Scorpius-Centaurus association), which additionally compresses the bubble walls.

\section{The Probable Birthplace of the Pulsar PSR B1929+10}

We considered the hypothesis of Hoogerwerf et al. (2001) that the star $\zeta$ Oph and the pulsar B1929+10 could be components of a binary in the neighborhood (within less than $10 \mathrm{pc}$ ) of US about 1 Myr ago using currently available data for the pulsar. Our simulations of encounters showed that, contrary to the view of Chatterjee et al. (2004), improved data for the pulsar only strengthen this hypothesis. Therefore, it seems most plausible. This requires 
that the pulsar radial velocity fall within the range $V_{r}=200 \pm 50 \mathrm{~km} \mathrm{~s}^{-1}$.

Our hypothesis of the HIP 86768 - B1929+10 binary, which could exist about 1 Myr ago in a wide neighborhood (within less than 80 pc) of IC4665, also seems plausible. The probability of the existence of such a binary is lower than that in the hypothesis of Hoogerwerf et al. (2001). A distinctly different pulsar radial velocity, $V_{r}=2 \pm 50 \mathrm{~km} \mathrm{~s}^{-1}$, is required for our hypothesis to hold.

An examination of the hypothesis by Walter (2001) about the $\zeta$ Oph-RXJ185635-3754 binary showed its probability to be an order of magnitude lower than that of our hypothesis.

\section{CONCLUSIONS}

Based on the epicyclic approximation, we simulated the motion of the young open star clusters IC 4665 and Collinder 359. The separation between the cluster centers was found to have been minimal $7 \mathrm{Myr}$ ago, 36 pc. This suggests that IC 4665 and Collinder 359 were formed from the same parent hydrogen cloud.

We showed a close evolutionary connection between IC 4665 and the Scorpius-Centaurus association. Thus, for example, the separation between the centers of these structures was $\approx 200$ pc about 15 Myr ago. At that time, IC 4665 was near two well-known regions of coronal gas: the Local Bubble and the North Polar Spur. This means that supernova explosions in IC 4665 over the past 15 Myr could affect the development of bubbles and, in particular, the North Polar Spur.

Analysis of the parameters for the encounter of the pulsar PSR B1929+10 with various stars in the vicinity of IC 4665 and Cr 359 suggests that the star HIP 86768 is a suitable candidate for a binary (in the past) with the pulsar. Monte Carlo simulations of the encounters of this pair with the pulsar radial velocity $V_{r}=2 \pm 50 \mathrm{~km} \mathrm{~s}^{-1}$ showed that out of the 3 million orbits, 22708 encounters occur at distances $\Delta_{r}<10 \mathrm{pc}(0.8 \%)$; in 6932 of the 22708 cases, the star and the pulsar were no farther than 80 pc from the center of IC 4665 about 1 Myr ago. We showed that using currently available data for the pulsar increases the probability of the hypothesis by Hoogerwerf et al. (2001) about the breakup of the $\zeta$ Oph-B1929+10 binary in the immediate vicinity of US about 1 Myr ago. Monte Carlo simulations of the encounters of this pair with the pulsar radial velocity $V_{r}=200 \pm 50 \mathrm{~km}$ $\mathrm{s}^{-1}$ showed that out of the 3 million orbits, 74115 encounters occur at distances $\Delta_{r}<10 \mathrm{pc}$ $(2.5 \%)$; in 5611 of the 74115 cases, the star and the pulsar were no farther than 10 pc from the US center about 1 Myr ago.

\section{ACKNOWLEDGMENTS}

I am grateful to A.T. Bajkova for help in the work and to the referees for several useful remarks that contributed to an improvement of the paper. This work was supported by the Russian Foundation for Basic Research (project nos. 05-02-17047 and 08-02-00400). 


\section{REFERENCES}

1. H. A. Abt and O. Cardona, Astrophys. J. 272, 182 (1983).

2. T.W. Berghöfer and D. Breitschwerdt, Astron. Astrophys. 390, 299 (2002).

3. A. Blaauw, Ann. Rev. Astron. Astrophys. 2, 213 (1964).

4. A. Blaauw, The Physics of star Formation and Early Stellar Evolution, Ed. by C. J. Lada and N. D. Kylafis (Kluwer, Dordrecht, 1991).

5. V. V. Bobylev, Pis'ma Astron. Zh. 30, 185 (2004) [Astron. Lett. 30, 159 (2004)].

6. V. V. Bobylev, Pis'ma Astron. Zh. 32, 906 (2006) [Astron. Lett. 32, 816 (2006)].

7. V. V. Bobylev and A. T. Bajkova, Pis'ma Astron. Zh. 33, 643 (2007) [Astron. Lett. 33, 571 (2007)].

8. D. Breitschwerdt and M. A. de Avillez, Astron. Astrophys. 452, L1 (2006).

9. W. F. Brisken, J. M. Benson, W. M. Goss, et al., Astrophys. J. 571, 906 (2002).

10. S. Chatterjee, J. M. Cordes, W. H. T. Vlemmings, et al., Astrophys. J. 604, 339 (2004).

11. W. Dehnen and J. J. Binney, Mon. Not. R. Astron. Soc. 298, 387 (1998).

12. D. Fernández, F. Figueras, and J. Torra, Highlights of Spanish Astrophysics IV Ed. by F. Figueras et al. (Springer-Verlag, New York, 2006); astro-ph: 0611766v1 (2006).

13. D. Fernández, F. Figueras, and J. Torra, astro-ph: 0801.0605v1 (2008).

14 . M. P. Fitzgerald, Astron. J. 73, 983 (1968).

15. P. C. Frish, Space Sci. Rev. 72, 499 (1995).

16. B. Fuchs, D. Breitschwerdt,M. A. Avilez, et al., Mon. Not. R. Astron. Soc. 373, 993 (2006).

17. E. J. de Geus, P. T. de Zeeuw, and J. Lub, Astron. Astrophys. 216, 44 (1989).

18. G. A. Gontcharov, Pis'ma Astron. Zh. 32, 844 (2006) [Astron. Lett. 32, 759 (2006)].

19. C. Heiles, Astrophys. J. 498, 689 (1998).

20. R. Hoogerwerf, J. H. J. de Bruijne, and P. T. de Zeeuw, Astron. Astrophys. 365, 49 (2001).

21. N. V. Kharchenko, R.-D. Scholz, A. E. Piskunov, et al., Astron. Nachr. 328 (2007).

22. P. G. Kulikovskii, Zvezdnaya astronomiya (Stellar Astronomy) (Nauka, Moscow, 1985) [in Russian].

23. B. Lindblad, Ark. Mat., Astron. Fys. 20 A, No. 17 (1927).

24. B. Lindblad, Handbuch der Physik 53, 21 (1959).

25. N. Lodieu, J. Bouvier, D. J. James, et al., Astron. Astrophys. 450, 147 (2006).

26. J. Maiz-Apellániz, Astrophys. J. 560, L83 (2001).

27. E. E. Mamajek, M. Meyer, and J. Liebert, Astron. J. 124, 1670 (2002).

28. S. Manzi, S. Randich, W. J. de Wit, et al., astro-ph/ 0712.0226v1 (2007).

29. J. C. Mermilliod, Astron. Astrophys. 97, 235 (1981).

30. E. D. Miller, H. Tsunemi, M. W. Bautz, et al., astro-ph/ 0708.4227v1 (2007).

31. C. Motch, A. M. Pires, F. Haberl, et al., Astrophys. Space Sci. 308, 217 (2006).

32. C. A. Olano, Astron. Astrophys. 121, 295 (2001).

33. V. G. Ortega, R. de la Reza, E. Jilinski, et al., Astrophys. J. 575, L75 (2002).

34 .C. A. Perrot and I. A. Grenier, Astron. Astrophys. 404, 519 (2003).

35. A. E. Piskunov, N. V. Kharchenko, S. Röser, et al., Astron. Astrophys. 445, 545 (2006).

36. S. B. Popov,M. Colpi,M. E. Prokhorov, et al., Astron. Astrophys. 406, 111 (2003).

37. T. Preibish and H. Zinnecker, Astron. J. 117, 2381 (1999).

38. C. F. Prosser, Astron. J. 105, 1441 (1993).

39. M. J. Sartori, J. R. D. Lépine, and W. S. Dias, Astron. Astrophys. 404, 913 (2003).

40. D. M. Sfeir, R. Lallement, F. Grifo, et al., Astron. Astrophys. 346, 785 (1999).

41. S. L. Snowden, R. Egger, M. J. Freyberg, et al., Astrophys. J. 485, 125 (1997).

42. The Hipparcos and Tycho Catalogues, ESA SP-1200, (1997). 
43. F.M.Walter, Astrophys. J. 549, 433 (2001).

44. R.Wielen, Astron. Astrophys. 13, 309 (1971).

45. R. Willingale, A. D. P. Hands, R. S. Warwick, et al., Mon. Not. R. Astron. Soc. 343, 995 (2003).

46. W. J. de Wit, J. Bouvier, F. Palla, et al., Astron. Astrophys. 448, 189 (2006).

47. P. T. de Zeeuw, R. Hoogerwerf, J. H. J. de Bruijne, et al., Astron. J. 117, 354 (1999). 
Table 1: Data on the objects

\begin{tabular}{|c|c|c|c|c|c|}
\hline Object & $\begin{array}{c}\alpha_{(J 2000.0)} \\
\delta_{(J 2000.0)}\end{array}$ & $\begin{array}{c}\mu_{\alpha} \cos \delta \\
\operatorname{mas} \mathrm{yr}^{-1}\end{array}$ & $\begin{array}{c}\mu_{\delta} \\
\text { mas } \mathrm{yr}^{-1}\end{array}$ & $\begin{array}{c}\pi \\
\text { mas }\end{array}$ & $\begin{array}{c}V_{r} \\
\mathrm{~km} \mathrm{~s}^{-1}\end{array}$ \\
\hline $\mathrm{B} 1929+10$ & $\begin{array}{c}19^{h} 32^{m} 13^{s} .9497 \\
10^{\circ} 59^{\prime} 32^{\prime \prime} .4203\end{array}$ & $94.09 \pm 0.11$ & $42.99 \pm 0.16$ & $2.77 \pm 0.07$ & - \\
\hline IC 4665 & $\begin{array}{c}17^{h} 46^{m} \\
5^{\circ} 43^{\prime}\end{array}$ & $-0.57 \pm 0.30$ & $-7.40 \pm 0.36$ & $2.84 \pm 0.56$ & $-16.0 \pm 1.1$ \\
\hline Cr 359 & $\begin{array}{c}18^{h} 01^{m} \\
2^{\circ} 54^{\prime}\end{array}$ & $0.22 \pm 0.28$ & $-8.90 \pm 0.26$ & $2.22 \pm 0.99$ & $-4.6 \pm 0.2$ \\
\hline HIP 66524 & $\begin{array}{l}13^{h} 38^{m} 09^{s} .0095 \\
-50^{\circ} 20^{\prime} 59^{\prime \prime} .702\end{array}$ & $-60.75 \pm 0.49$ & $-13.34 \pm 0.67$ & $2.28 \pm 0.75$ & $23 \pm 1$ \\
\hline $\begin{array}{l}\mathrm{HIP} 81377 \\
(\zeta \mathrm{Oph})\end{array}$ & $\begin{array}{l}16^{h} 37^{m} 09^{s} .5378 \\
-10^{\circ} 34^{\prime} 01^{\prime \prime} .524\end{array}$ & $13.07 \pm 0.85$ & $25.44 \pm 0.72$ & $7.12 \pm 0.71$ & $-9.9 \pm 5.5$ \\
\hline HIP 86768 & $\begin{array}{c}17^{h} 43^{m} 47^{s} .0205 \\
-7^{\circ} 04^{\prime} 46^{\prime \prime} .588\end{array}$ & $-7.12 \pm 0.70$ & $-10.39 \pm 0.51$ & $2.34 \pm 0.80$ & $19.0 \pm 4.3$ \\
\hline HIP 91599 & $\begin{array}{c}18^{h} 40^{m} 48^{s} .0517 \\
-8^{\circ} 43^{\prime} 07^{\prime \prime} .688\end{array}$ & $-9.64 \pm 1.13$ & $-22.64 \pm 0.79$ & $3.61 \pm 1.16$ & $29 \pm 5$ \\
\hline RXJ185635-3754 & $\begin{array}{l}18^{h} 56^{m} 35^{s} .56 \\
-37^{\circ} 54^{\prime} 37^{\prime \prime} .0 \\
\end{array}$ & $326.7 \pm 0.8$ & $-59.1 \pm 0.7$ & $16.5 \pm 2.3$ & - \\
\hline
\end{tabular}

Table 2: Positions and heliocentric velocities of the objects

\begin{tabular}{|c|c|c|c|c|c|c|}
\hline & $\begin{array}{c}X(0), \\
\mathrm{pc}\end{array}$ & $\begin{array}{c}Y(0), \\
\mathrm{pc}\end{array}$ & $\begin{array}{c}Z(0), \\
\mathrm{pc}\end{array}$ & $\begin{array}{c}U(0), \\
\mathrm{km} \mathrm{s}^{-1}\end{array}$ & $\begin{array}{c}V(0), \\
\mathrm{km} \mathrm{s}^{-1}\end{array}$ & $\begin{array}{c}W(0), \\
\mathrm{km} \mathrm{s}^{-1}\end{array}$ \\
\hline B1929+10 & $244 \pm 6$ & $265 \pm 7$ & $-25 \pm 1$ & $-109 \pm 34$ & $91 \pm 37$ & $-107 \pm 6$ \\
$\mathrm{IC} 4665$ & $290 \pm 57$ & $171 \pm 34$ & $104 \pm 21$ & $-6 \pm 2$ & $-17 \pm 3$ & $-9 \pm 2$ \\
Cr 359 & $381 \pm 169$ & $217 \pm 96$ & $99 \pm 44$ & $6 \pm 8$ & $-16 \pm 14$ & $-10 \pm 8$ \\
HIP 66524 & $279 \pm 92$ & $-326 \pm 107$ & $90 \pm 30$ & $-83 \pm 67$ & $-102 \pm 57$ & $1 \pm 3$ \\
HIP 81377 & $128 \pm 13$ & $14 \pm 1$ & $56 \pm 6$ & $-11 \pm 5$ & $18 \pm 2$ & $-1 \pm 2$ \\
HIP 86768 & $397 \pm 136$ & $134 \pm 46$ & $86 \pm 29$ & $25 \pm 7$ & $-18 \pm 17$ & $6 \pm 2$ \\
HIP 91599 & $253 \pm 81$ & $113 \pm 36$ & $-8 \pm 3$ & $39 \pm 7$ & $-18 \pm 13$ & $-3 \pm 1$ \\
RxJ185635-3754 & $58 \pm 8$ & $-1.4 \pm 0.2$ & $-18 \pm 3$ & $-75 \pm 48$ & $20 \pm 1$ & $-75 \pm 15$ \\
\hline
\end{tabular}

Note. The velocities of the pulsar B1929+10 were calculated for its radial velocity $V_{r}=2 \pm 50 \mathrm{~km}$ $\mathrm{s}^{-1}$; the velocities of the pulsar RX J185635.3754were calculated for $V_{r}=-50 \pm 50 \mathrm{~km} \mathrm{~s}^{-1}$. 

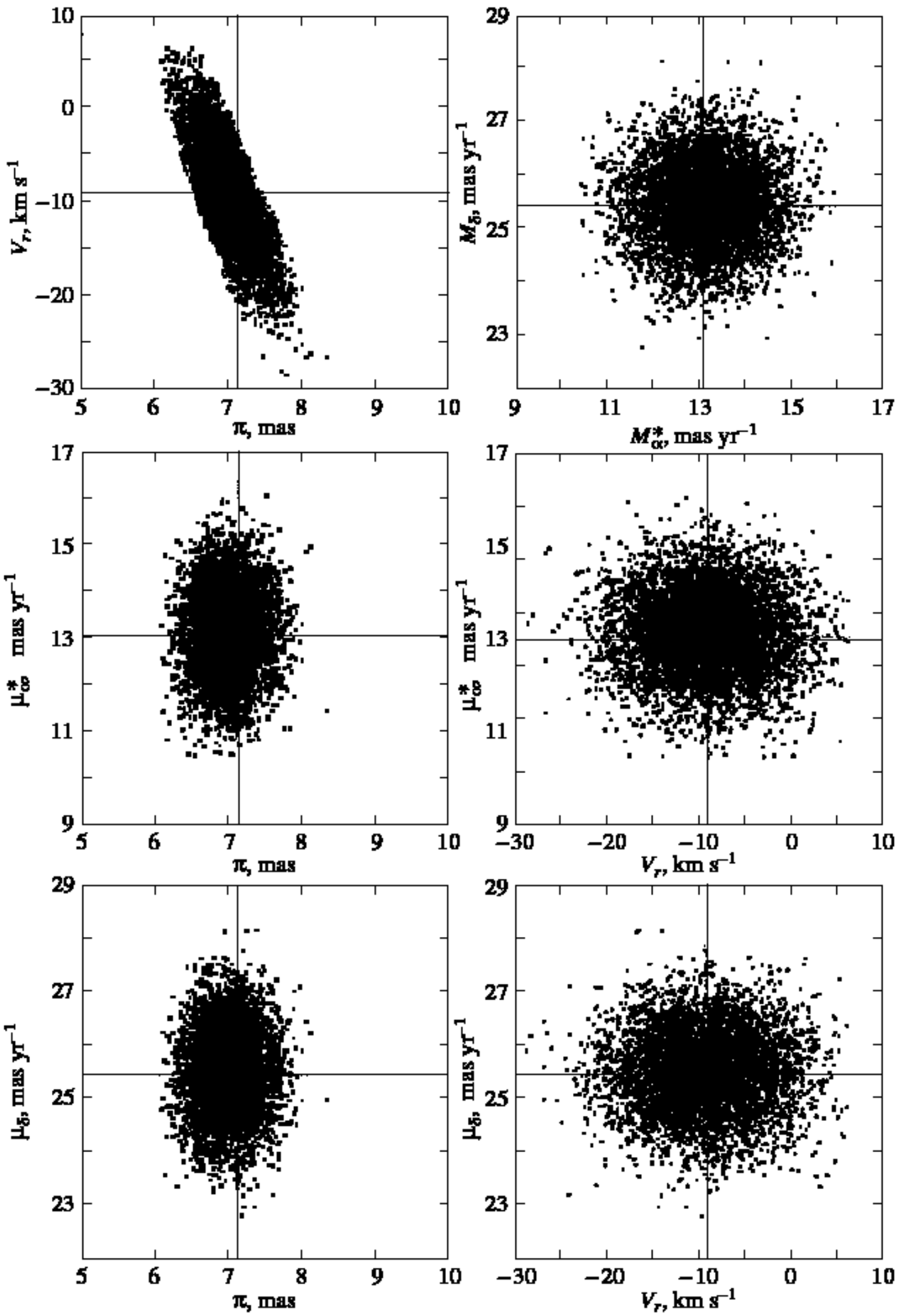

Fig. 1. Domains of admissible values at which 5611 encounters occur at distances $\Delta_{r}<10 \mathrm{pc}$ between the star $\zeta$ Oph and the pulsar PSR B1929+10, during which they fall into the neighborhood of US, $\Delta_{r}<10 \mathrm{pc}$ for $\zeta$ Oph. 

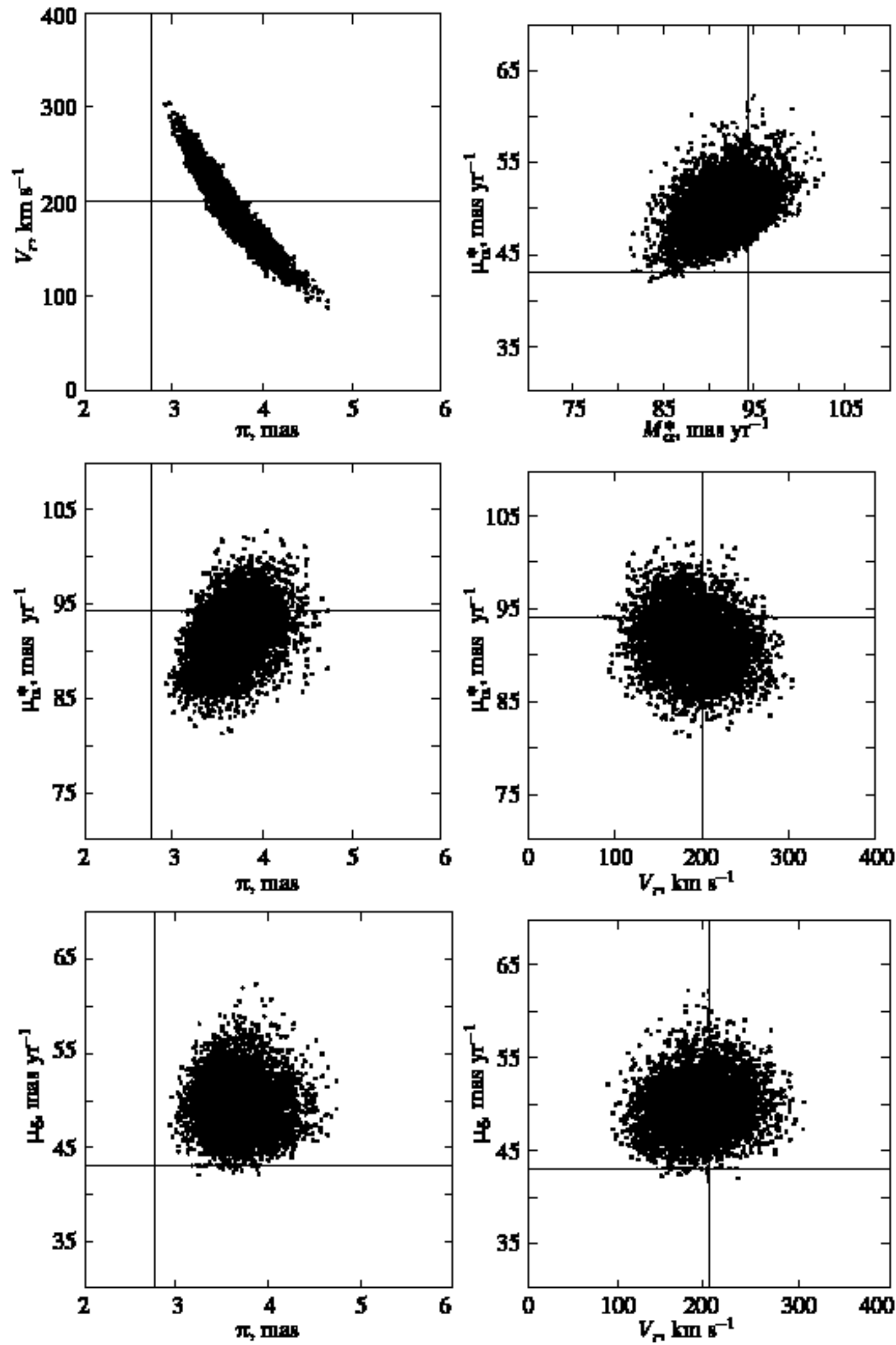

Fig. 2. Domains of admissible values at which 5611 encounters occur at distances $\Delta_{r}<10 \mathrm{pc}$ between the star $\zeta$ Oph and the pulsar PSR B1929+10, during which they fall into the neighborhood of US, $\Delta_{r}<10$ pc for PSR B1929+10. 

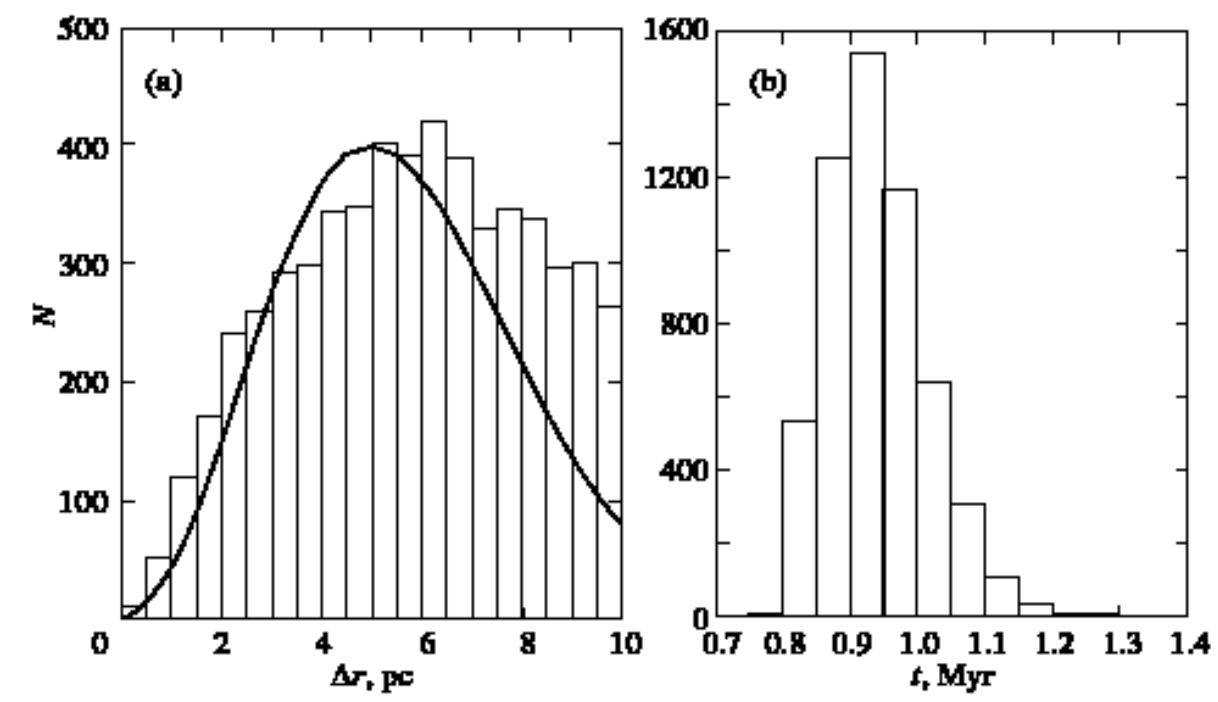

Fig. 3. (a) Expected distribution of minimum distance $\Delta_{r}<10$ pc for 5611 encounters of $\zeta$ Oph with PSR B1929+10 and (b) histogram of encounter times. 

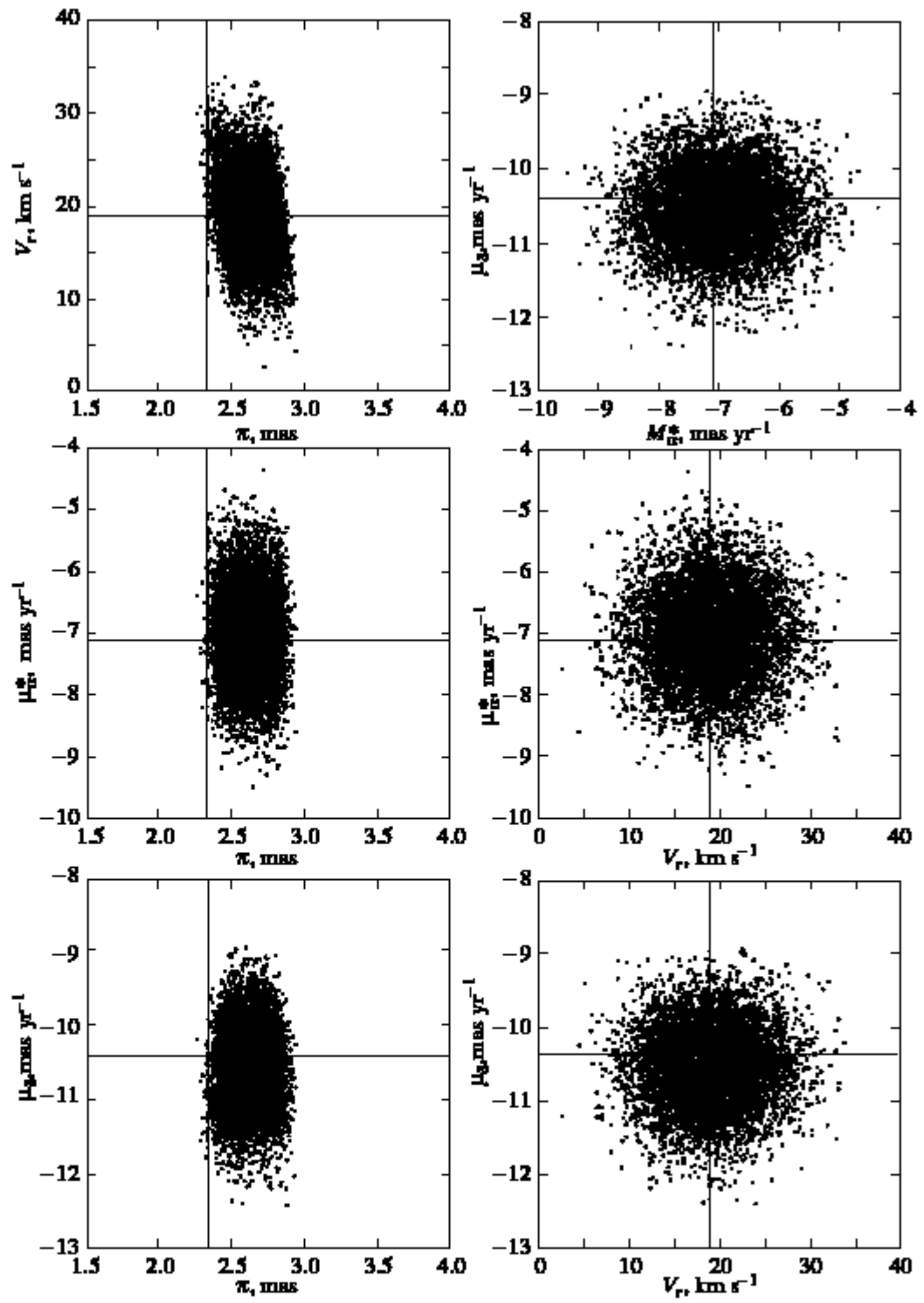

Fig. 4. Domain of admissible values at which 6932 encounters occur at distances $\Delta_{r}<10 \mathrm{pc}$ between HIP 86768 and PSR B1929+10, during which they fall into the neighborhood of IC $4665, \Delta_{r}<80$ pc for HIP 86768. 

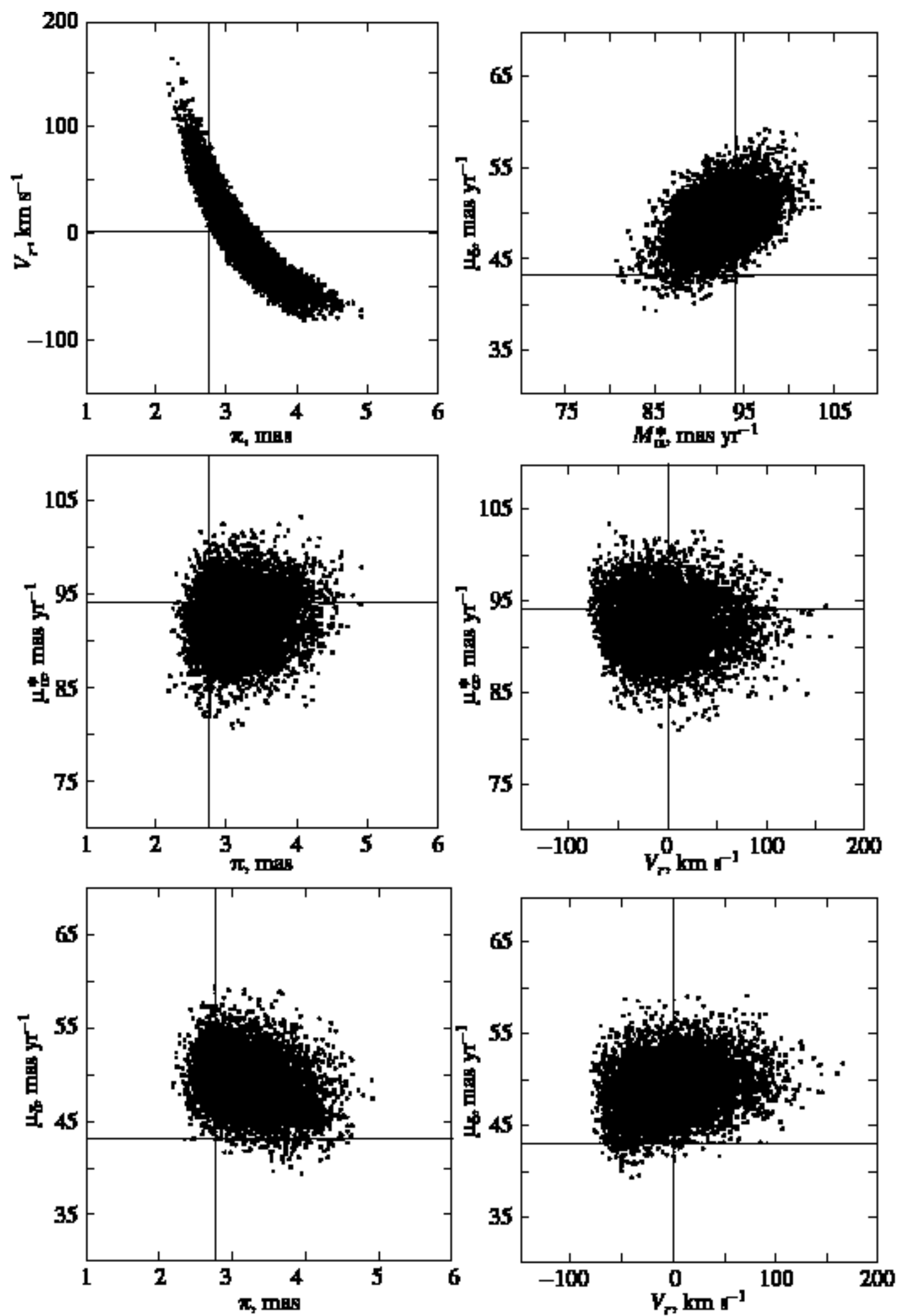

Fig. 5. Domains of admissible values at which 6932 encounters occur at distances $\Delta_{r}<10 \mathrm{pc}$ between HIP 86768 and PSR B1929+10, during which they fall into the neighborhood of IC $4665, \Delta_{r}<80$ pc for PSR B1929+10. 

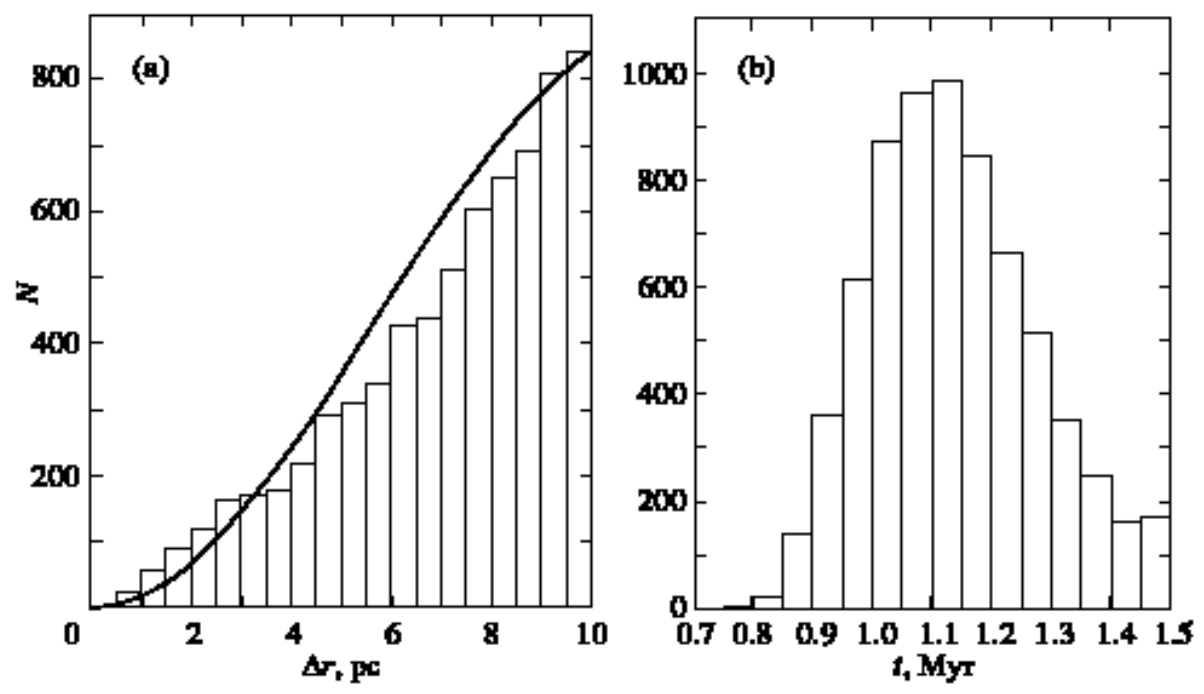

Fig. 6. (a) Expected distribution of minimum distance $\Delta_{r}<10$ pc for 6932 encounters of HIP 86768 with PSR B1929+10 and (b) histogram of encounter times. 


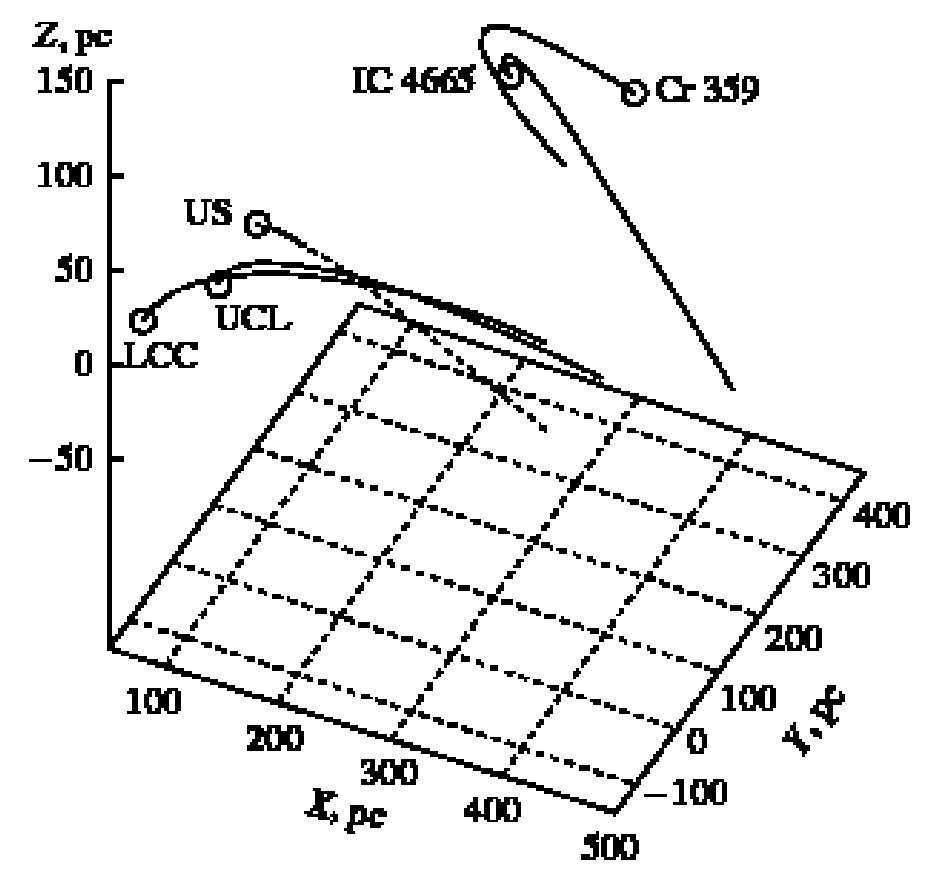

Fig. 7. Spatial coordinates of IC 4665, Cr 359, members of the Scorpius-Centaurus association, and their trajectories in the past $30 \mathrm{Myr}$.

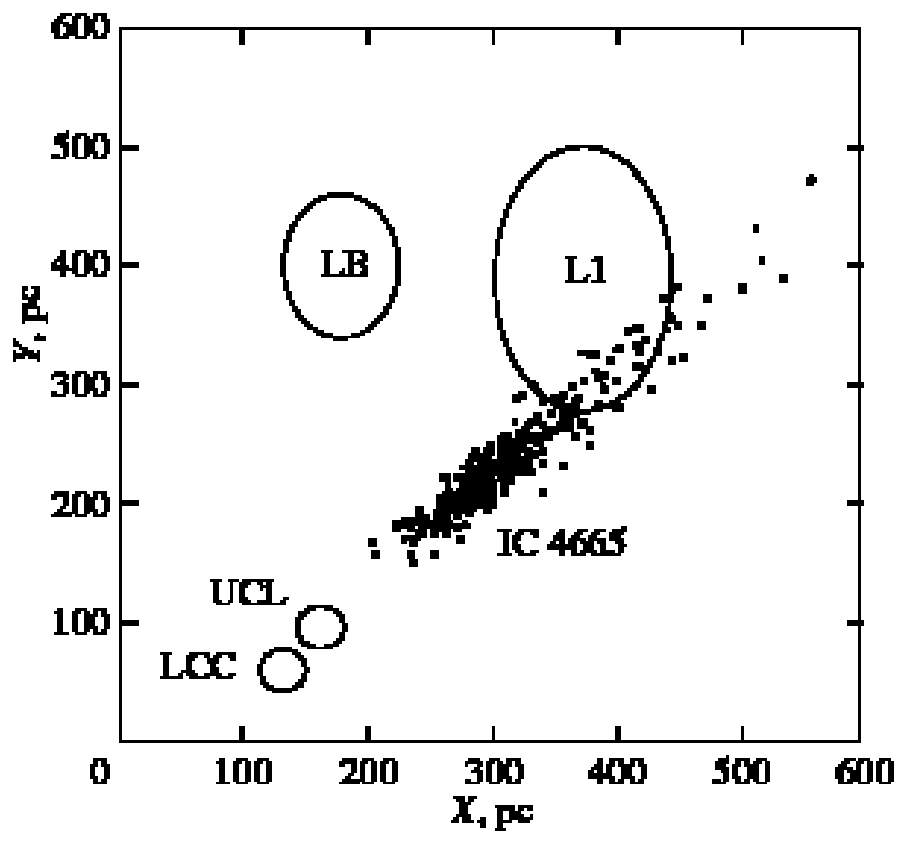

Fig. 8. Positions of IC 4665, UCL, LCC, the Local Bubble (LB), and the North Polar Spur (L1) (Breitschwerdt and de Avillez 2006) 15 Myr ago.. 